\title{
BMJ Open Effect of medicines management versus standard care on readmissions in multimorbid patients: a randomised controlled trial
}

\author{
Marianne Lea (D) , ${ }^{1}$ Morten Mowé, ${ }^{2,3}$ Espen Molden,, ${ }^{4,5}$ Kristin Kvernrød, \\ Eva Skovlund, ${ }^{6}$ Liv Mathiesen ${ }^{4,7}$
}

To cite: Lea M, Mowé $M$, Molden $\mathrm{E}$, et al. Effect of medicines management versus standard care on readmissions in multimorbid patients: a randomised controlled trial. BMJ Open 2020;10:e041558. doi:10.1136/ bmjopen-2020-041558

- Prepublication history for this paper is available online. To view these files, please visit the journal online (http://dx.doi. org/10.1136/bmjopen-2020041558).

Received 11 June 2020 Revised 23 November 2020 Accepted 02 December 2020

D Check for updates

(c) Author(s) (or their employer(s)) 2021. Re-use permitted under CC BY-NC. No commercial re-use. See rights and permissions. Published by BMJ.

For numbered affiliations see end of article.

Correspondence to Dr Marianne Lea; marianne.lea@ sykehusapotekene.no

\section{ABSTRACT}

Objective To investigate the effect of pharmacist-led medicines management in multimorbid, hospitalised patients on long-term hospital readmissions and survival.

Design Parallel-group, randomised controlled trial. Setting Recruitment from an internal medicine hospital ward in 0slo, Norway. Patients were enrolled consecutively from August 2014 to the predetermined target number of 400 patients. The last participant was enrolled March 2016. Follow-up until 31 December 2017, that is, 21-40 months.

Participants Acutely admitted multimorbid patients $\geq 18$ years, using minimum four regular drugs from minimum two therapeutic classes. 399 patients were randomly assigned, 1:1, to the intervention or control group. After excluding 11 patients dying in-hospital and 2 erroneously included, the primary analysis comprised 386 patients (193 in each group) with median age 79 years (range 23-96) and number of diseases 7 (range 2-17). Intervention Intervention patients received pharmacistled medicines management comprising medicines reconciliation at admission, repeated medicines reviews throughout the stay and medicines reconciliation and tailored information at discharge, according to the integrated medicines management model. Control patients received standard care.

Primary and secondary outcome measures The primary endpoint was difference in time to readmission or death within 12 months. Overall survival was a priori the clinically most important secondary endpoint.

Results Pharmacist-led medicines management had no significant effect on the primary endpoint time to readmission or death within 12 months (median 116 vs 184 days, HR $0.82,95 \% \mathrm{Cl} 0.64$ to $1.04, \mathrm{p}=0.106$ ). A statistically significantly increased overall survival was observed during 21-40 months follow-up (HR 0.66, 95\% $\mathrm{Cl} 0.48$ to $0.90, \mathrm{p}=0.008$ ).

Conclusions Pharmacist-led medicines management had no statistically significant effect on time until readmission or death. A statistically significant increased overall survival was seen. Further studies should be conducted to investigate the effect of such an intervention on a larger scale.

Trial registration number NCT02336113.
Strengths and limitations of this study

- Randomised controlled design, blinded in the steps possible to blind.

- Included almost 200 high-risk multimorbid patients in each group and followed them for 20-41 months.

- Hard endpoints, readmissions and mortality, collected from national registers.

Inclusion from a single hospital in Norway.

- Spillover effect may have reduced the effect estimate.

\section{INTRODUCTION}

Increased life expectancy and steadily improving healthcare contribute to a growing subpopulation of multimorbid patients, commonly defined as having a minimum of two conditions. ${ }^{1-3}$ The prevalence of multimorbidity is reported to be $20 \%-30 \%$ in the general population, $55 \%-98 \%$ in the elderly and $22 \%-65 \%$ in hospitalised patients. ${ }^{4-6}$ Multimorbidity is associated with the use of multiple drugs, increased use of healthcare services and reduced life expectancy. ${ }^{37-9}$ The organisation of healthcare services and treatment guidelines is, however, mainly focused on single diagnoses, while coexisting diagnoses or use of multiple drugs are rarely taken into account. ${ }^{10}{ }^{11}$ Studying the care of multimorbid patients is crucial to managing the future global challenge of ensuring safe, effective and evidence-based care to these patients. ${ }^{11112}$

Multimorbid patients using numerous drugs are at high risk of harm by drug-related problems (DRPs). ${ }^{13} 14$ DRPs are reported to cause $10 \%-30 \%$ of all hospital admissions, whereof a high proportion is preventable. ${ }^{15-17}$ Drugs also cause problems during the hospital stay, ${ }^{18} 19$ which pose a risk of readmissions. ${ }^{2021}$ A recent Cochrane review found no evidence that medicines reviews reduce hospital readmissions or mortality. ${ }^{22}$ The authors state 
that important effects may have been overlooked due to short follow-up in included studies, and request highquality studies with long follow-up in high-risk patient populations. $^{22}$

The integrated medicines management (IMM) model has been established as a tool for clinical pharmacists to optimise and individualise drug therapy. ${ }^{23}$ IMM comprises a systematic approach to ensure high-quality of the use of drugs throughout the hospital stay, comprising a three-step procedure, that is, medicines reconciliation at admission, medicines reviews during the stay and medicines reconciliation and information at discharge. ${ }^{23-27}$ Nevertheless, only a very limited number of clinical pharmacists are working in Norwegian hospitals, hence standard care for hospitalised patients does not include IMM or other services by clinical pharmacists. Several studies have investigated the effect of implementing either parts of, or the complete IMM model on different efficacy measures, ${ }^{23-25} 28$ but to our knowledge, not in multimorbid patients. The objective of the present study was to investigate the effect of pharmacist-led medicines management in multimorbid, hospitalised patients on long-term hospital readmissions and survival.

\section{MATERIALS AND METHODS \\ Study design}

This parallel-group, randomised controlled trial was conducted at the internal medicine ward, Oslo University hospital (Ullevaal), Norway. The ward comprised 24 beds and mainly received patients with multiple medical issues, in particular haematological, endocrine, infectious and/ or cardiovascular. Patients were considered for inclusion Monday to Friday during regular daytime working hours, from 30 August 2014, until the predetermined target number of 400 patients was enrolled. Eligible patients were prospectively invited and enrolled in the study following written informed consent. Online supplementary appendix S1 shows the original trial protocol, protocol amendments, the statistical analysis plan and the timeline of the study with the milestones. Online supplementary appendix S2 shows the Consolidated Standards of Reporting Trials checklist. Figure 1 gives a graphical depiction of the study design, as suggested for studies of complex interventions. ${ }^{29}$

The trial was registered in ClinicalTrials.gov, identifier: NCT02336113, in June 2014. Due to a minor Protocol Registration and Results System review comment, the trial was first published on their website in January 2015. A clarification that readmission data were to be harvested from the Norwegian Patient Registry, was the only addition to the original registration. The trial is closed for new participants.

\section{Participants}

Inclusion criteria were: acute admission, age $\geq 18$ years and use of at least four regular drugs from minimum two therapy classes (Anatomical Therapeutic Chemical ${ }^{30}$ at first level) at admission. The latter was chosen as the preferred multimorbidity measure, ${ }^{31}$ as drug counts were considered more reliable than disease counts in the acute hospital admission setting. Drugs were counted before medicines reconciliation. However, if the medicines reconciliation revealed that this inclusion criterion was not fulfilled, the patient was excluded from the study. Exclusion criteria were (1) terminally ill, (2) isolated due to severe infections or (3) unable to communicate in Norwegian or English and no translator available. Patients readmitted during the study period were not invited for 'a second' inclusion.

\section{Randomisation and blinding}

The patients were randomised 1:1 to the intervention or control group. Centre for Biostatistics and Epidemiology, Oslo University Hospital, was responsible for the randomisation procedure. Their staff had no contact with patients, study pharmacists or ward staff. A random number generator programme and a permuted block design were used to generate the randomisation sequence, which was delivered to the study pharmacists in sequentially numbered, opaque, sealed envelopes. The investigators were blinded to block size, which was randomly varied. Randomisation took place following patient inclusion and baseline assessments. A study pharmacist assigned the envelope with the lowest number to the individual participant and signed the allocation before the envelope was opened.

It was neither feasible to blind participants nor study pharmacists to the allocation. It was also known by ward staff which of the patients belonged to the intervention group. Ward staff was, however, unable to distinguish between patients randomised to the control group and patients not participating in the trial. The primary endpoint analysis was conducted on a blinded dataset (by researchers who did not see patients). The staff from the Norwegian Patient Registry and the Norwegian Cause of Death Registry providing outcome data were not involved in data collection or preparation of data files and were blinded to group allocation.

\section{Data collection and baseline assessments}

During the inclusion period, six clinical pharmacists, all with a master's degree in clinical pharmacy and standardised training in IMM, collected data, conducted baseline assessments and provided the various steps of the intervention. All steps were standardised using translated IMM procedures adapted to the Norwegian hospital setting. ${ }^{23-27} 32$ A DRP was defined according to the Pharmaceutical Care Network Europe as 'an event or circumstance involving drug therapy that actually or potentially interferes with desired health outcomes' ${ }^{33}$

Blood samples were collected for biochemical analyses. Glomerular filtration rate was calculated using the Cockcroft-Gault formula, ${ }^{34}$ except for obese patients (body-mass index $>30$ ), for whom the Salazar-Corcoran formula was used. ${ }^{35}$ An experienced senior physician retrospectively collected information from medical 

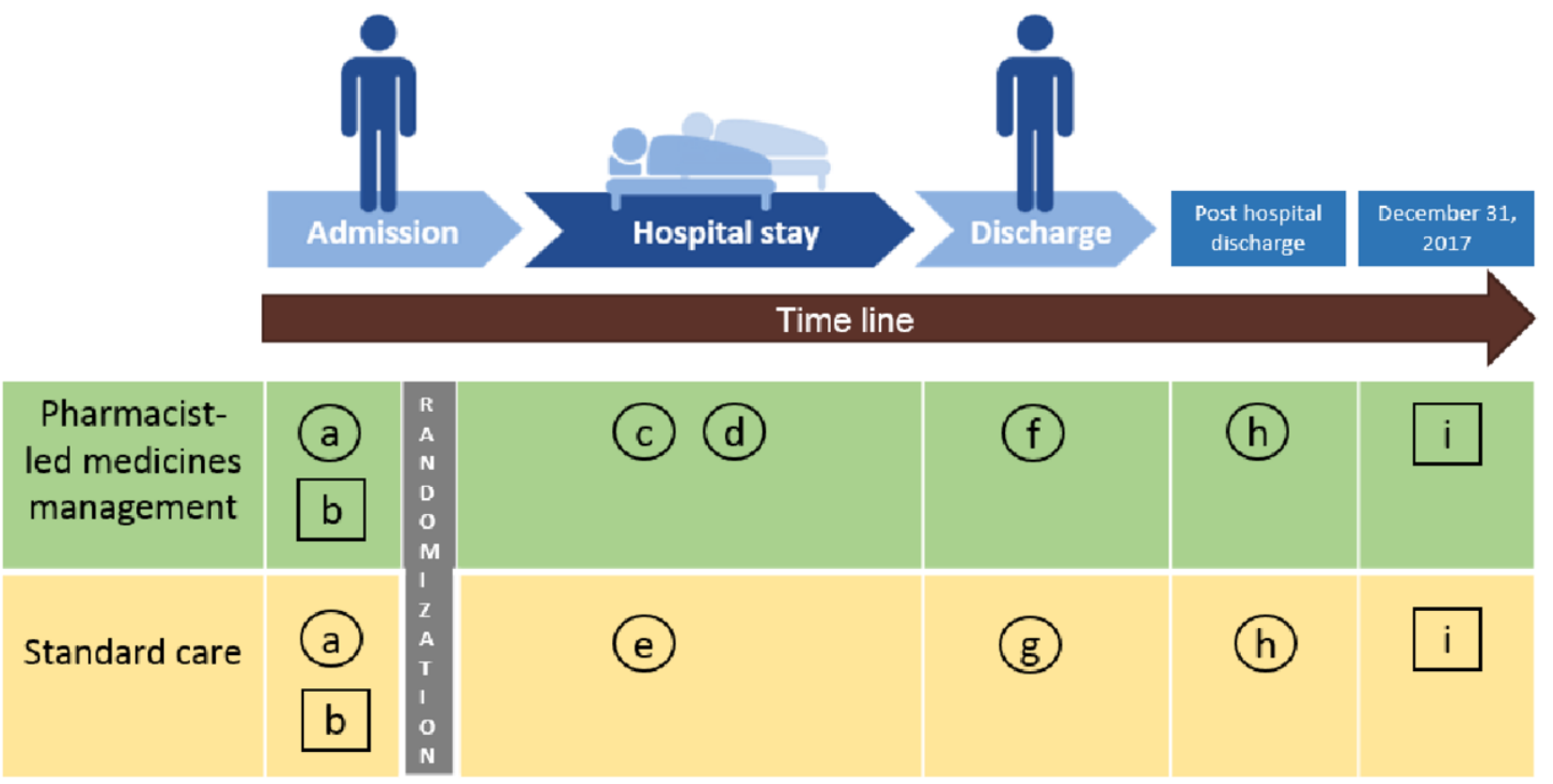

\begin{tabular}{|c|c|}
\hline a) & $\begin{array}{l}\text { Medicines reconciliation and review conducted by clinical pharmacist without discussion of medicine discrepancies or } \\
\text { drug-related problems (DRPs) and possible solutions in the multidisciplinary teama }\end{array}$ \\
\hline $\mathrm{b}$ & Patient characteristics collected \\
\hline & $\begin{array}{l}\text { Multidisciplinary treatment teama discussions of medicine discrepancies and DRPs revealed at hospital admission and } \\
\text { possible solutions }\end{array}$ \\
\hline & $\begin{array}{l}\text { Medicines review conducted by clinical pharmacists by systematically addressing } 11 \text { pre-defined risk categories for } \\
\text { each drug each patient was prescribed at a given point of time; drug monitoring, adverse effect, drug-drug interaction, } \\
\text { non-optimal drug therapy, reduced organ function/contraindication, inappropriate drug in elderly, unnecessary drug, } \\
\text { course length, practical problem, adherence issue and other. Medicines reviews were performed at admission and } \\
\text { repeatedly as needed due to changes in either prescription, patient symptoms, clinical state, and/or laboratory values. } \\
\text { Patients were reviewed for such changes daily, Monday to Friday, during regular daytime working hours. Consecutively } \\
\text { multidisciplinary treatment teama discussions of identified DRPs and possible solutions. }\end{array}$ \\
\hline & $\begin{array}{l}\text { Standard in-hospital care provided by physicians with internal medicine expertize, nursing staff and when needed; } \\
\text { clinical nutrition physiologists and/or physiotherapists }\end{array}$ \\
\hline & $\begin{array}{l}\text { Medicines reconciliation, followed by written and verbal information tailored to the patient's further needs of care, as } \\
\text { well as discharge activities aiming to ensure continuous treatment and increase adherence: } \\
\text { - Written systematic information comprising a reconciled drug list with description and justification for all changes } \\
\text { made during the hospital stay, to the next care provider (all patients), and to the patient/relative if they to some } \\
\text { extent would be involved in handling the drugs after discharge } \\
\text { - Verbal information/conversation with the patient and/or relative adapted to the patient needs }{ }^{c} \text { - if they to some } \\
\text { extent would be involved in handling the drugs after discharge } \\
\text { - Assistance with retrieving drugs from the pharmacy, if needed } \\
\text { - Providing the patient with drugs from the hospital pending on an updated multidose delivery, if needed }\end{array}$ \\
\hline & $\begin{array}{l}\text { Discharge medicine information (not standardized) provided by physicians with internal medicine expertize and } \\
\text { nursing staff }\end{array}$ \\
\hline & Standard care in the primary health care (details not collected) \\
\hline & Last day of follow-up on readmissions and mortality outcomes \\
\hline
\end{tabular}

a The multidisciplinary treatment team consisted of physician with expertise in internal medicine, nursing staff, clinical pharmacist, and when needed; clinical nutrition physiologists and/or physiotherapists

${ }^{\mathrm{b}}$ The general practitioner, nursing home, home nurse and/or multidose delivering pharmacy.

c Sometimes regarding the entire drug list, sometimes the most important changes, sometimes regarding one specific drug. If the patient was considered to benefit from any provided information or given the opportunity to ask questions, such a targeted conversation was conducted, even if a complex conversation was not considered favorable.

Figure 1 Graphical depiction of the study design, inspired by Perera et al. ${ }^{29}$ Objects are represented by squares and activities by circles. 
records to calculate the Charlson Comorbidity Index (CCI) score. ${ }^{36}$

Before allocation, baseline assessments were conducted for all included patients, comprising medicines reconciliation and review. The purpose of these baseline assessments was to assess the prevalence of DRPs and drug-related hospitalisations. ${ }^{37}$ These medicines reviews included only drugs used before admission, not drugs initiated during transport, or following hospital admission. The pharmacists had access to the patient's medical history and laboratory results up to and including admission time. Importantly though, medicines discrepancies, that is, mismatches between the reconciled drug list and the list recorded at hospital admission, and DRPs revealed during these baseline assessments were neither discussed in the multidisciplinary treatment team, nor documented in the patient record. Before allocation, the study pharmacist assessed whether any medicines discrepancy or DRP could result in irreversible detrimental effects or death if not handled immediately. If the patient was allocated to the control group, any such issue was discussed with a senior physician (MM) who decided whether it was necessary to intervene.

\section{The intervention group: in-hospital pharmacist-led medicines management}

The thorough intervention implied the inclusion of clinical pharmacist(s) in the patients' multidisciplinary treatment team throughout the hospital stay, working in close collaboration with the patient, physicians and other members of the team, as shown in figure 1 . The medicines management process can be divided into three parts covering the patients' hospital stay; medicines reconciliation at admission, medicines review repeatedly during the entire stay and medicines reconciliation and tailored information at discharge. ${ }^{23-27}$ Medicines reviews were performed at admission and repeatedly as needed due to changes in either prescription, patient symptoms, clinical state and/or laboratory values. Patients were reviewed for such changes daily, Monday to Friday, during regular daytime working hours.

During medicines reviews, a list of predefined risk categories, all described in detail in table 1, were systematically addressed for each drug in each patient. Furthermore, an overall benefit-risk assessment was made with the main goal of tailoring drug therapy to the individual participant, giving significant weight to the patient perspective. Medicines discrepancies and DRPs revealed during both baseline assessments and the hospital stay were discussed in the multidisciplinary treatment team. At discharge, a medicines reconciliation was conducted, followed by written and oral information tailored to the patient's further needs of care, provided to the patient and/or next care provider, see figure 1 . The main goals of this step were to answer drug questions, to ensure continuous treatment, to increase adherence, and to provide the patient and/or next care provider a complete overview of all drugs.

\section{The control group: standard care}

The control group received standard care, see figure 1, which in line with standard procedures in Norwegian hospitals included neither medicines reconciliation nor medicines reviews or any other service from clinical pharmacists. Medicines discrepancies and DRPs revealed during baseline assessments in control patients were only registered in the research database, and not discussed in the multidisciplinary treatment team.

\section{Endpoints}

The primary endpoint was time to first hospital readmission or death within 12 months after discharge.

Secondary endpoints:

- Overall survival.

- Number of unplanned hospitalisations per patient within 12 months after discharge.

- Proportion of patients:

- With unplanned hospitalisations within 30 days, 6 months and 12 months after discharge.

- Who died within 30 days, 6 months, 12 months and 20 months after discharge.

- Who died or had unplanned hospitalisations within 30 days, 6 months and 12 months after discharge.

- Length of stay (LOS) of first hospital readmission.

- Time to the first unplanned readmission within 12 months after discharge, censored for deaths.

In the original trial protocol, included in online supplementary appendix S1, the difference between the control and intervention group in time to the first readmission was defined as the primary endpoint without further specification. As death is a competing risk to readmissions, it was considered appropriate to use the difference in time to readmission or death as the primary endpoint. This was clarified in the statistical analysis plan, which was finalised and signed before outcome data files were available.

Data on readmissions were provided by the Norwegian Patient Registry and data on mortality by the Norwegian Cause of Death Registry. We had originally planned a follow-up of 12 months. However, as both the inclusion period and the retrieval of outcome data took longer than planned, we decided to extend the follow-up of all patients to 31 December 2017, to increase statistical power. This amendment was described in the statistical analysis plan, which was finalised and signed before any outcome data files were available. Because the inclusion period lasted approximately 1.5 years, the follow-up of each individual patient was in the range 21-40 months.

The primary efficacy analysis was a modified intention to treat-analysis excluding patients who died during the index hospital stay as they were never at risk for readmission, as well as erroneously included patients. The analysis population was defined before outcome data files were received.

\section{Sample size}

The sample size calculation was based on an expected 12 -month readmission frequency of $50 \%{ }^{23}$ It was 


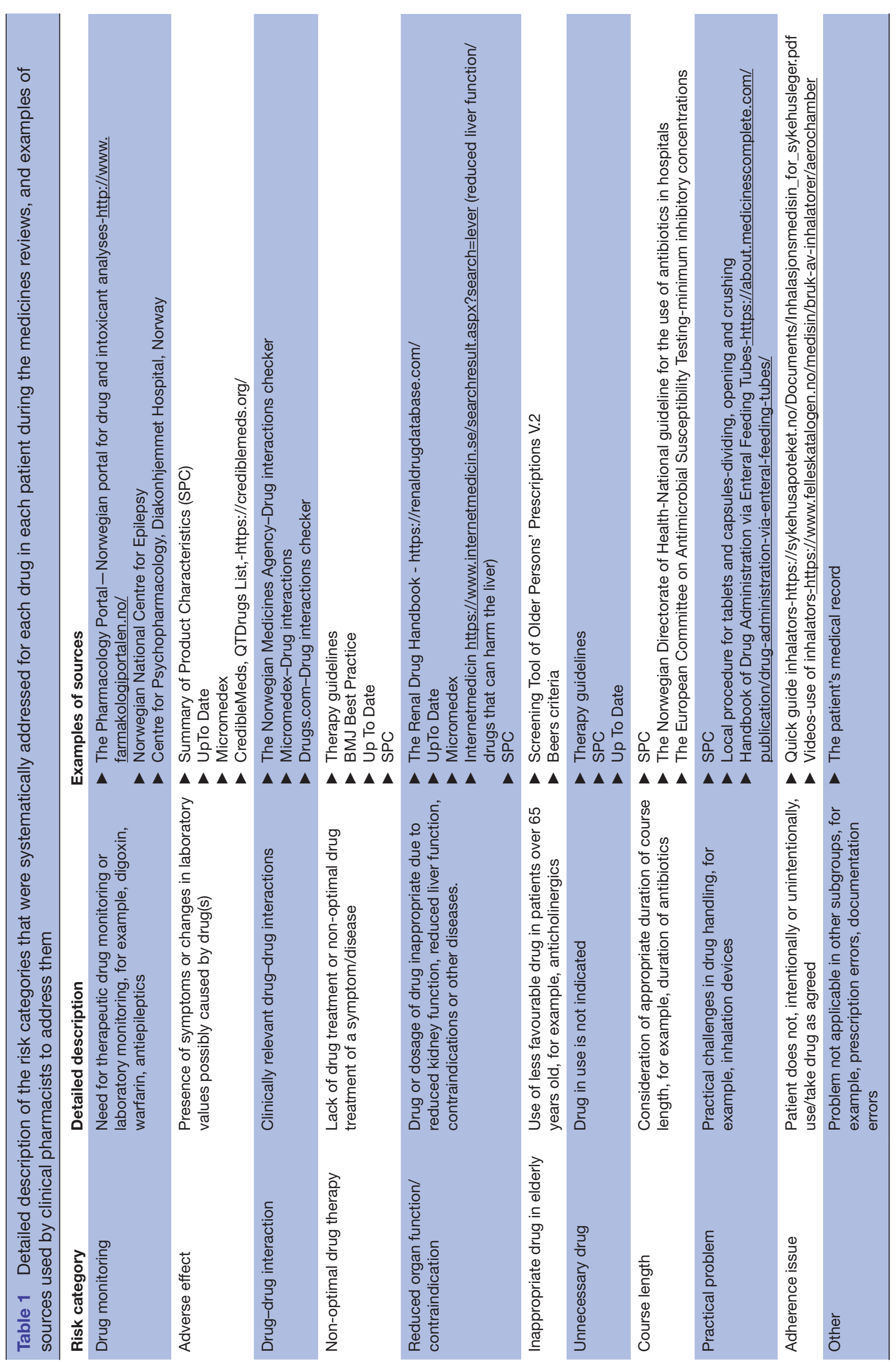


estimated that to detect a $15 \%$ absolute reduction in hospital readmissions with $80 \%$ power and a significance level of 5\%, we would need 168 patients in each group. To compensate for any dropouts, it was decided to enrol 200 patients in each group. Sample size calculations based on proportions are generally considered reliable for survival analysis, but might in some instances overestimate the required sample size. ${ }^{38}$ In other words: since a survival analysis utilises the information better than a comparison of proportions at a given time, the power will be somewhat higher than estimated above.

\section{Statistics}

Time-to-event endpoints were compared between groups by the Kaplan-Meier method and the log-rank test. Cox's proportional hazards model was applied to estimate hazard ratios (HRs), which are presented with 95\% confidence intervals (CIs). The proportionality assumption was checked by visual inspection of $\log (-\log )$ plots. Continuous variables were compared between the two groups using Mann-Whitney tests. In an additional sensitivity analysis of time to readmission, which was not included in the statistical analysis plan, death was treated as a competing risk using the Fine and Grey method. ${ }^{39}$

Statistical analyses were performed by IBM SPSS Software version 25.0 (IBM Corp. NY) and STATA 16. P values $<0.05$ were regarded as statistically significant.

\section{Patient and public involvement}

During the planning of the study, patient representatives from the medical clinic participated in the preparation of the patient information leaflet and provided input on the study design, for example, the choice of the primary endpoint.

\section{RESULTS}

During the study period, 30 August 2014 to 17 March 2016, 2174 patients were admitted to the internal medicine ward and 1769 (81\%) were assessed for eligibility. Figure 2 shows the patient flow. Among the 598 patients invited to participate, 175 (29\%) declined (permission to register reasons for declining not obtained). A total of 399 patients were randomised, 200 to the intervention group and 199 to the control group. Following randomisation, 11 patients (5 intervention and 6 control) who died during the hospital stay and two patients (both intervention) who were erroneously included, were excluded from the analyses. Thus, the analysis population for all endpoints comprised 193 patients in each group, all followed-up until 31 December 2017, that is, for a minimum of 21 months and a maximum of 40 months.

The median age in the analysis population was 79 years (range 23-96), 356 (92\%) were home dwelling before hospitalisation and 213 (55\%) were women. The median number of regular drugs at hospital admission was 8 (range 4-19). The median number of diseases was 7 (range 2-17) and the median CCI score was 3 (range 0-12). The median number of DRPs per patient identified during baseline assessments was 13 (range 3-42). The baseline characteristics of the patients in the control versus the intervention group are presented in table 2. No differences of importance were observed between the groups.

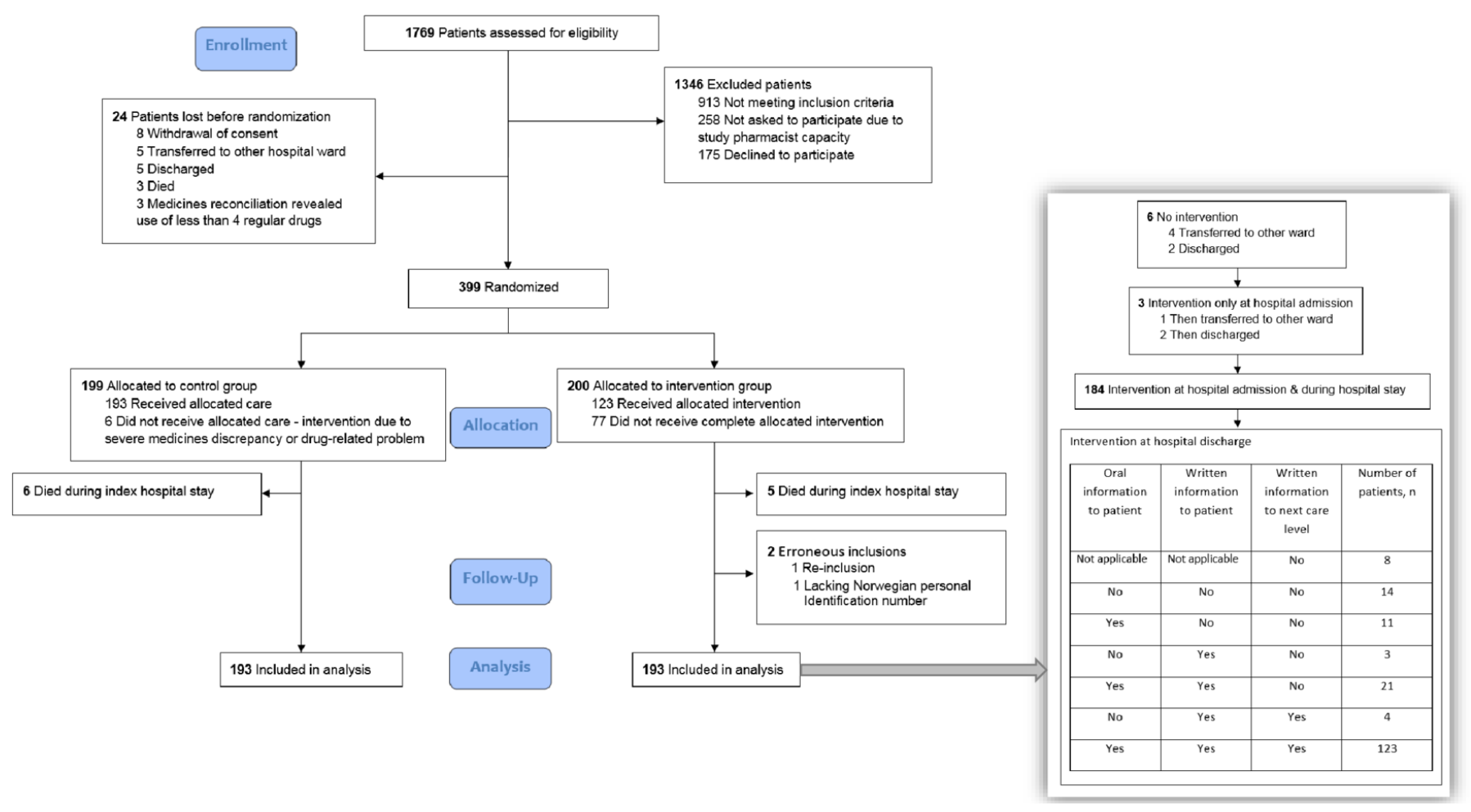

Figure 2 Patient flow. 
Table 2 Characteristics of patients in the analysis population

\begin{tabular}{|c|c|c|}
\hline Characteristic & Control (n=193) & Intervention $(n=193)$ \\
\hline Women & $106(55 \%)$ & $102(53 \%)$ \\
\hline Age & $80.7(23.1-96.4)$ & $78.0(25.7-95.6)$ \\
\hline Charlson Comorbidity Index score & $3(0-12)$ & $2(0-11)$ \\
\hline \multicolumn{3}{|l|}{ Most frequent medical history: } \\
\hline - Endocrine and metabolic diseases & $77(40 \%)$ & $80(42 \%)$ \\
\hline Kidney disease & $63(33 \%)$ & $73(38 \%)$ \\
\hline Congestive heart failure & $81(42 \%)$ & $68(35 \%)$ \\
\hline - Arrhythmia & $72(37 \%)$ & $71(37 \%)$ \\
\hline Body mass index ${ }^{\star}$ & $24.4(14.4-48.4)$ & $25.0(13.1-43.3)$ \\
\hline - Serum albumin $(\mathrm{g} / \mathrm{L}) \dagger$ & $38(24-51)$ & $38(22-56)$ \\
\hline - C reactive protein (nmol/L) & $133(0-3419)$ & $152(0-5248)$ \\
\hline \multicolumn{3}{|l|}{ No of prescribed drugsł at hospital admission: } \\
\hline - Regular & $8(4-19)$ & $8(4-19)$ \\
\hline - On demand & $2(0-10)$ & $2(0-11)$ \\
\hline \multicolumn{3}{|c|}{ Assistance with drug administration before hospitalisation: } \\
\hline - Multidose & $51(26 \%)$ & $46(24 \%)$ \\
\hline - Home nurse & $33(17 \%)$ & $28(15 \%)$ \\
\hline Nursing home & $15(8 \%)$ & $15(8 \%)$ \\
\hline \multicolumn{3}{|l|}{ Assistance with drug administration after discharge: } \\
\hline - Multidose & $28(15 \%)$ & $26(14 \%)$ \\
\hline Home nurse & $32(17 \%)$ & $21(11 \%)$ \\
\hline Nursing home & $51(26 \%)$ & $51(26 \%)$ \\
\hline - Relative & 7 (4\%) & $11(6 \%)$ \\
\hline Other institution/hospital ward & $18(9 \%)$ & $13(7 \%)$ \\
\hline
\end{tabular}

Data are $\mathrm{n}(\%)$ or median (range).

*Body mass index was registered for $144 / 193$ control patients and 148/193 intervention patients.

†Serum albumin was registered for 181/193 control patients and 187/193 intervention patients.

$\ddagger$ After medicines reconciliation.

In the group receiving pharmacist-led medicines management, a total of 3826 DRPs were revealed at hospital admission and during the hospital stay. Type of DRPs revealed and presented for discussion in the multidisciplinary team and the respective acceptance rates will be presented in a separate publication. In overall numbers, 1100 of the 3826 identified DRPs (29 $\%$ ) were solved without the need for discussion in the multidisciplinary treatment team, while 1075 (28\%) were not prioritised for discussion, that is, considered of low importance compared with other DRPs or the patients' clinical state. The remaining 1651 (43\%) DRPs were discussed in the multidisciplinary team, whereof $1022(62 \%)$ led to immediate changes in the individual patient's drug treatment. In 6 of the 193 control patients $(1.5 \%)$ severe medicines discrepancies or DRPs that 

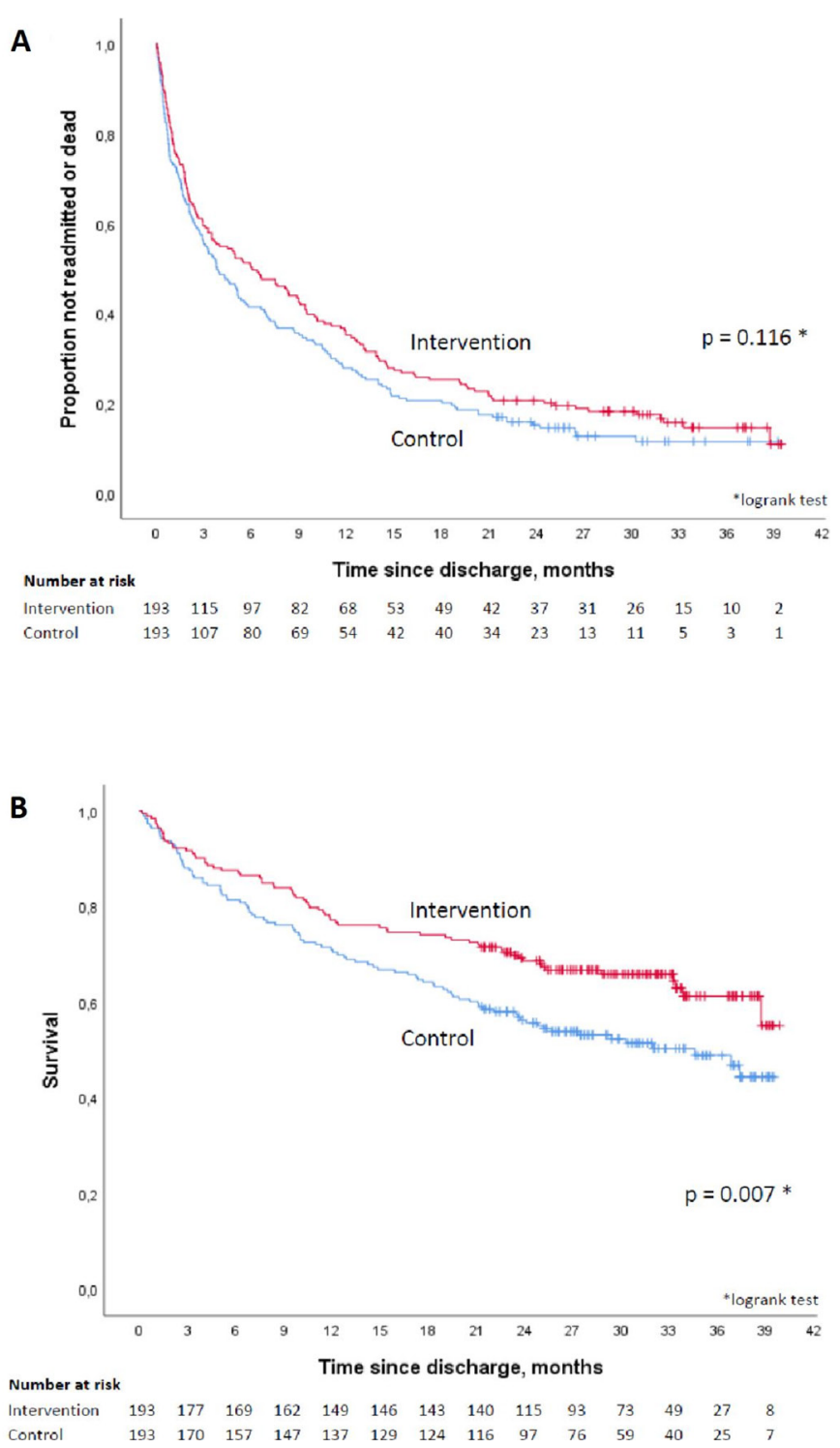

Figure 3 (A) Time to first hospital readmission or death in the intervention versus the control group. (B) Overall survival in the intervention versus the control group.

had to be intervened on were revealed during baseline assessments.

Figure 3A shows time to first readmission or death in the two groups. The median time to readmission or death was 184 days in the intervention group and 116 days in the control group, but the difference was not statistically significant (HR 0.82, 95\% CI 0.64 to $1.04, \mathrm{p}=0.106$ ). Sensitivity analyses, extending follow-up until 31 December 2017 , or excluding control patients who were intervened on, did not influence the effect estimate (HR 0.84, 95\% CI 0.68 to $1.05, p=0.118$ and HR $0.85,95 \%$ CI 0.68 to 1.06 , $\mathrm{p}=0.149$, respectively). The secondary endpoint analysis of time to first readmission, censoring for 20 deaths, gave a similar effect estimate (HR $0.81,95 \%$ CI 0.63 to 1.04 , $\mathrm{p}=0.104$ ), shown in online supplementary appendix S3. When death was instead treated as a competing risk the subdistribution HR was SHR 0.83, 95\% CI 0.64 to 1.06 , $\mathrm{p}=0.137$.
There was a statistically significant difference in overall survival (HR 0.66, 95\% CI 0.48 to $0.90, \mathrm{p}=0.008$ ), as shown in figure 3B. The results of other the secondary endpoint analyses are shown in table 3 . Within 20 months after the index discharge, $27 \%$ of the intervention patients had died vs $39 \%$ of the control patients.

\section{DISCUSSION}

Pharmacist-led medicines management in multimorbid patients did not statistically significantly prolong the time until first readmission or death compared with control patients. The result is in contrast with previous randomised controlled trials (RCTs) on similar interventions provided to other patient populations, showing a decreased readmission rate, prolonged time to readmission, and a reduction in hospital visits. ${ }^{23} 40-42$ This contrast may be explained by the patient population. To our knowledge, our study is the first to investigate the effect of a medicines management intervention on clinically relevant endpoints in multimorbid patients with complex drug regimens. In this population, urgent medical care like hospital readmissions might be difficult to avoid. This theory is supported by a subgroup analysis of one of the previous RCTs, which found that in patients 80 years or older a pharmacist intervention was more effective in preventing emergency department visits in patients using less than five drugs compared with patients using five drugs or more. ${ }^{28}$ However, it should be noted that the $95 \%$ CI in our study is wide and compatible with a risk reduction of $36 \%$ as well as a $4 \%$ increased risk. The sample size calculation in the current study was based on a target $15 \%$ reduction in readmissions, which may have been optimistic, and insufficient power may therefore explain the non-significant result.

A statistically significantly increased overall survival, one of the secondary endpoints, was seen in patients in the intervention vs the control group. The hazard reduction of $34 \%$ is indisputably clinically relevant and reflects a great improvement potential in the care of multimorbid patients. To our knowledge, this is the first study to show an effect of pharmacist-led medicines management on survival. This endpoint was either not investigated, ${ }^{23} 42$ or no effect was seen ${ }^{40}$ in the previous RCTs. The results of our study are in contrast to the recent Cochrane review concluding that 'medication review does not seem to prevent death and hospital readmissions'. ${ }^{22}$ The reason for this discrepancy is most likely multifactorial and due to differences in patient populations, characteristics of the interventions, and the duration of the follow-up. Important differences in the patient populations include older patients in the study by Gillespie et $a l^{40}$ and that the study by Ravn-Nielsen $e t a l^{43}$ included patients with lower mortality than the current study, that is, mortality rates of $10 \%$ vs $19 \%$, respectively, in the control group at 6 months after index discharge. In our study, a thorough intervention conducted close to the patient, including medicines reconciliation both at admission and discharge as well as 


\begin{tabular}{|c|c|c|c|}
\hline Endpoint & $\begin{array}{l}\text { Intervention group } \\
(\mathrm{n}=193)\end{array}$ & $\begin{array}{l}\text { Control group } \\
(n=193)\end{array}$ & $P$ value \\
\hline $\begin{array}{l}\text { No of unplanned hospitalisations per patient within } 12 \text { months } \\
\text { after discharge, median (range) }\end{array}$ & $1(0-13)$ & $1(0-12)$ & 0.212 \\
\hline $\begin{array}{l}\text { Length of hospital stay of first unplanned hospitalisation, median } \\
\text { no of days (range) }\end{array}$ & $6(1-58)$ & $6(1-71)$ & 0.576 \\
\hline \multicolumn{4}{|l|}{ No of patients unplanned hospitalised within } \\
\hline - 6 months after index discharge, $\mathrm{n}(\%)$ & $89(46)$ & $103(53)$ & 0.154 \\
\hline - 12 months after index discharge, $\mathrm{n}(\%)$ & $115(60)$ & $129(67)$ & 0.139 \\
\hline \multicolumn{4}{|l|}{ No of patients who died within } \\
\hline - 30 days after index discharge, n (\%) & $4(2)$ & $7(4)$ & 0.359 \\
\hline \multicolumn{4}{|l|}{ No of patients who died or was unplanned hospitalised within } \\
\hline - 30 days after index discharge, $\mathrm{n}(\%)$ & $41(21)$ & $51(26)$ & 0.232 \\
\hline - 6 months after index discharge, $\mathrm{n}(\%)$ & $96(50)$ & $113(59)$ & 0.082 \\
\hline - 12 months after index discharge, $\mathrm{n}(\%)$ & $125(65)$ & $139(72)$ & 0.125 \\
\hline
\end{tabular}

improved information at discharge to ensure continuous treatment and increase adherence, may constitute characteristics of the intervention important for the effect on survival. Clinical pharmacists performing the procedures of the intervention in close collaboration with the patient, physician and other members of the treatment team are most likely also important for obtaining the effect on survival. At last, the longer follow-up in the present study, prolonged by several months compared with the other RCTs, ${ }^{40} 43$ could have allowed prophylactic drugs added during medicine reviews enough time to achieve beneficial effects ${ }^{22}$ and probably contributes to explain the intervention's effect on survival.

Heterogeneity in the pharmacist-led in-hospital interventions, including various elements of various intensity, make comparisons of results among studies, as well as interpretation of results, challenging. ${ }^{22} 44$ Furthermore, such interventions are indisputably complex, and evaluating such interventions is complicated. ${ }^{45} 46$ The intervention consists of various components delivered as an overall intervention. With such a design, it is not known whether the overall intervention or only parts of it are important for effect. The intervention in the current study consisted of elements of the highest level of intensity, that is, diamond-level medicines reconciliation ${ }^{44} 47$ and advanced medicines reviews. ${ }^{48}$ In the recent RCT from Denmark, a similar intervention of similar intensity reduced emergency department visits and hospital readmissions but did not have an effect on mortality, ${ }^{43}$ that is, the opposite of our results. Differences in eligibility criteria, nuances in the delivered intervention and/or care delivered to control patients, clinical pharmacists' training and how they interacted with the rest of the multidisciplinary treatment team may be factors contributing to explain this. The current study nevertheless adds to the international body of literature that high-intensity, in-hospital pharmacist-led interventions to tailor drug therapy may improve clinical outcomes in high-risk patients.

The intervention had no effect on the LOS of the first readmission. This was not surprising, as hospitals in Norway for several years have received incentives to reduce LOS, illustrated by as short as 6 days median LOS of the first readmission in the present study. In comparison, an IMM-intervention showed a reduction from 13.1 days to 9.7 days LOS of the first readmission in Northern Ireland. ${ }^{23}$ The number of unplanned hospitalisations during 12 months follow-up did not differ between the groups in the present study, in line with findings by Gillespie et $a t^{40}$

Drug counts were chosen as the preferred multimorbidity measure at patient inclusion, which could be seen as a limitation. Nonetheless, this strategy resulted in the inclusion of a multimorbid patient population, as validated by diseases counts according to the generally accepted definition. ${ }^{3}$ Our study included patients from a single hospital in Norway which may challenge the generalisability. However, the study had few exclusion criteria, thus comprising a broad population. The low drop-out rate further contributes favourably to external validity.

It was not feasible to blind participants, study pharmacists or ward physicians to group allocation. To limit bias, the study was blinded on all steps considered possible to blind. Any spillover effect of the intervention to control patients would, in any case, reduce the effect estimate. Due to the complexity of the intervention a proportion of the intervention patients did not receive the complete 
intervention, which may also have contributed to the non-significance on the primary endpoint and an underestimation of the effect on survival. The broad inclusion criteria may have resulted in the inclusion of participants at low risk of readmission and death, which might also have contributed to the non-significant result on the primary endpoint, as well as buffered the effect of the intervention on survival. Studying the effect of pharmacist-led medicines management in a subgroup of multimorbid patients at the highest risk of readmission, for example, by stratifying on frailty, could be useful. The randomised controlled design and the long follow-up of all patients are factors that strengthen the study.

\section{CONCLUSION}

Pharmacist-led medicines management in-hospital to multimorbid patients had no statistically significant effect on time until readmission or death. A statistically significant increase in overall survival was seen. As a response to the increasing challenges of providing safe and evidencebased healthcare to high-risk multimorbid patients, further studies should be conducted to investigate the effect of such an intervention on a larger scale.

\section{Author affiliations}

'Department of Pharmaceutical Services, Oslo Hospital Pharmacy, Hospital Pharmacies Enterprise, South Eastern Norway, Oslo, Norway

${ }^{2}$ General Internal Medicine Ward, the Medical Clinic, Oslo University Hospital, Oslo, Norway

${ }^{3}$ Institute of Clinical Medicine, University of Oslo, Oslo, Norway

${ }^{4}$ Department of Pharmacy, Section for Pharmacology and Pharmaceutical

Biosciences, University of Oslo, Oslo, Norway

${ }^{5}$ Center for Psychopharmacology, Diakonhjemmet Hospital, Oslo, Norway

${ }^{6}$ Department of Public Health and Nursing, Norwegian University of Science and Technology, NTNU, Trondheim, Norway

${ }^{7}$ Hospital Pharmacies Enterprise, South Eastern Norway, Oslo, Norway

Correction notice This article has been corrected since it was published. DOI link has been included in the prepublication history. The provenance and peer review statement has been included.

Acknowledgements The authors thank the study pharmacists Anne Schwinghammer, Anette Engnes, Elin Trapnes, Hanne Steen and Petra Foynland for their valuable contribution in patient inclusion, medicines reconciliation and review, senior physician Jo Fuglestved for summarising the CCI scores, Anne Mette Njaastad, Kristin Hestad Solheim, Kristin Thomassen and Torhild Heggestad for valuable input on the study design, employees at the internal medicine ward for the positive attitude to the study, and finally Dominic Anthony Hoff for valuable support regarding data punching.

Contributors ML: conceptualisation, formal analysis, funding acquisition, investigation, methodology, project administration, software, writing —original draft, writing—review and editing. MM: conceptualisation, funding acquisition, methodology, project administration, supervision, writing — review and editing. EM: conceptualisation, funding acquisition, methodology, supervision, writing — review and editing. KK: investigation, methodology, resources, writing — review and editing ES: conceptualisation, formal analysis, funding acquisition, methodology, writing review and editing. LM: conceptualisation, formal analysis, funding acquisition, methodology, project administration, supervision, writing —original draft, writingreview and editing.

Funding This work was supported by South-Eastern Norway Regional Health Authority (Ph.D. grant number 12/00718 to author ML). Additional support was provided by the Hospital Pharmacies Enterprise and Oslo University Hospital and Diakonhjemmet hospital.
Disclaimer The funders had no role in study design, data collection and analysis, decision to publish, or preparation of the manuscript.Data from the Norwegian Patient Registry has been used in this publication. The interpretation and reporting of these data are the sole responsibility of the authors, and no endorsement by the Norwegian Patient Registry is intended nor should be inferred.

Competing interests ML received Ph.D. funding from the South-Eastern Norway Regional Health Authority (grant number 12/00718).

Patient consent for publication Not required.

Ethics approval The trial was approved by the Regional Committee for Medical and Health Research Ethics (2014/704/REK south-eastern D) and the Privacy Ombudsman at Oslo University Hospital.

Provenance and peer review Not commissioned; externally peer reviewed.

Data availability statement Data are available on reasonable request. Data may be obtained from a third party and are not publicly available. The data that support the findings of this study are available from Oslo University Hospital but restrictions apply to the availability of these data, which were used under license for the current study, and so are not publicly available. Deidentified participant data are however available from the authors on reasonable request and with permission of 0slo University Hospital, with publication. Additional related documents, for example, patient consent forms, are available at request.

Supplemental material This content has been supplied by the author(s). It has not been vetted by BMJ Publishing Group Limited (BMJ) and may not have been peer-reviewed. Any opinions or recommendations discussed are solely those of the author(s) and are not endorsed by BMJ. BMJ disclaims all liability and responsibility arising from any reliance placed on the content. Where the content includes any translated material, BMJ does not warrant the accuracy and reliability of the translations (including but not limited to local regulations, clinical guidelines, terminology, drug names and drug dosages), and is not responsible for any error and/or omissions arising from translation and adaptation or otherwise.

Open access This is an open access article distributed in accordance with the Creative Commons Attribution Non Commercial (CC BY-NC 4.0) license, which permits others to distribute, remix, adapt, build upon this work non-commercially, and license their derivative works on different terms, provided the original work is properly cited, appropriate credit is given, any changes made indicated, and the use is non-commercial. See: http://creativecommons.org/licenses/by-nc/4.0/.

\section{ORCID iD}

Marianne Lea http://orcid.org/0000-0001-8583-911X

\section{REFERENCES}

1 Uijen AA, van de Lisdonk EH. Multimorbidity in primary care: prevalence and trend over the last 20 years. Eur $\mathrm{J}$ Gen Pract 2008:14:28-32.

2 Jurevicienė E, Onder G, Visockienẻ Ž, et al. Does multimorbidity still remain a matter of the elderly: Lithuanian national data analysis. Health Policy 2018:122:681-6.

3 Mercer S, Salisbury C, Fortin M. Abc of multimorbidity. first ED. John Wiley \& Sons, 2014.

4 Marengoni A, Angleman S, Melis R, et al. Aging with multimorbidity: a systematic review of the literature. Ageing Res Rev 2011;10:430-9.

5 Roberts KC, Rao DP, Bennett TL, et al. Prevalence and patterns of chronic disease multimorbidity and associated determinants in Canada. Health Promot Chronic Dis Prev Can 2015;35:87-94.

6 Brett T, Arnold-Reed DE, Popescu A, et al. Multimorbidity in patients attending 2 Australian primary care practices. Ann Fam Med 2013;11:535-42.

7 Nobili A, Marengoni A, Tettamanti M, et al. Association between clusters of diseases and polypharmacy in hospitalized elderly patients: results from the REPOSI study. Eur J Intern Med 2011;22:597-602.

8 DuGoff EH, Canudas-Romo V, Buttorff C, et al. Multiple chronic conditions and life expectancy: a life table analysis. Med Care 2014;52:688-94.

9 Lehnert T, Heider D, Leicht $\mathrm{H}$, et al. Review: health care utilization and costs of elderly persons with multiple chronic conditions. Med Care Res Rev 2011;68:387-420.

10 Boyd CM, Darer J, Boult C, et al. Clinical practice guidelines and quality of care for older patients with multiple comorbid diseases: implications for pay for performance. JAMA 2005;294:716-24.

11 Tinetti ME, Fried TR, Boyd CM. Designing health care for the most common chronic condition--multimorbidity. JAMA 2012;307:2493-4. 
12 Barnett K, Mercer SW, Norbury M, et al. Epidemiology of multimorbidity and implications for health care, research, and medical education: a cross-sectional study. Lancet 2012;380:37-43.

13 Köberlein-Neu J, Mennemann H, Hamacher S, et al. Interprofessional medication management in patients with multiple morbidities. Dtsch Arztebl Int 2016;113:741-8.

14 Fiß T, Meinke-Franze C, van den Berg N, et al. Effects of a three Party healthcare network on the incidence levels of drug related problems. Int J Clin Pharm 2013;35:763-71.

15 Al Hamid A, Ghaleb M, Aljadhey H, et al. A systematic review of hospitalization resulting from medicine-related problems in adult patients. Br J Clin Pharmacol 2014;78:202-17.

16 Gustafsson M, Sjölander M, Pfister B, et al. Drug-Related hospital admissions among old people with dementia. Eur J Clin Pharmacol 2016;72:1143-53.

17 Rafter N, Hickey A, Conroy RM, et al. The Irish national adverse events study (INAES): the frequency and nature of adverse events in Irish hospitals-a retrospective record review study. BMJ Qual Saf 2017;26:111-9.

18 Lea M, Rognan SE, Koristovic R, et al. Severity and management of drug-drug interactions in acute geriatric patients. Drugs Aging 2013;30:721-7.

19 Hohmann C, Neumann-Haefelin T, Klotz JM, et al. Drug-Related problems in patients with ischemic stroke in hospital. Int J Clin Pharm 2012;34:828-31.

20 El Morabet N, Uitvlugt EB, van den Bemt BJF, et al. Prevalence and preventability of drug-related Hospital readmissions: a systematic review. J Am Geriatr Soc 2018;66:602-8.

21 Schwab C, Korb-Savoldelli V, Escudie JB, et al. latrogenic risk factors associated with Hospital readmission of elderly patients: a matched case-control study using a clinical data Warehouse. J Clin Pharm Ther 2018;43:393-400.

22 Christensen M, Lundh A. Medication review in hospitalised patients to reduce morbidity and mortality. Cochrane Database Syst Rev 2016;2:Cd008986.

23 Scullin C, Scott MG, Hogg A, et al. An innovative approach to integrated medicines management. J Eval Clin Pract 2007;13:781-8.

24 Midlöv P, Holmdahl L, Eriksson T, et al. Medication report reduces number of medication errors when elderly patients are discharged from hospital. Pharm World Sci 2008;30:92-8.

25 Scullin C, Hogg A, Luo R, et al. Integrated medicines management - can routine implementation improve quality? J Eval Clin Pract 2012;18:807-15.

26 Midlöv P, Deierborg E, Holmdahl L, et al. Clinical outcomes from the use of medication report when elderly patients are discharged from hospital. Pharm World Sci 2008;30:840-5.

27 Bergkvist A, Midlöv $P$, Höglund $P$, et al. Improved quality in the hospital discharge summary reduces medication errors--LIMM: Landskrona Integrated Medicines Management. Eur J Clin Pharmacol 2009;65:1037-46.

28 Alassaad A, Bertilsson M, Gillespie U, et al. The effects of pharmacist intervention on emergency department visits in patients 80 years and older: subgroup analyses by number of prescribed drugs and appropriate prescribing. PLoS One 2014;9:e111797.

29 Perera R, Heneghan C, Yudkin P. Graphical method for depicting randomised trials of complex interventions. BMJ 2007;334:127-9.

30 World Health Organization. Collaborating centre for drug statistics methodology. ATC index with DDDs. Available: https://www.whocc. no/atc_ddd_index/[Accessed 20 Sep 2018].
31 Wallace E, McDowell R, Bennett K, et al. Comparison of count-based multimorbidity measures in predicting emergency admission and functional decline in older community-dwelling adults: a prospective cohort study. BMJ Open 2016;6:e013089.

32 Nilsson N, Lea M, Lao Y, et al. Medication discrepancies revealed by medication reconciliation and their potential short-term and long-term effects: a Norwegian multicentre study carried out on internal medicine wards. European Journal of Hospital Pharmacy 2015;22:298-303.

33 Parmaceutical Care Network Europe (PCNE). Classification for drug related problems V 6.2. Available: http://www.pcne.org/ upload/files/11_PCNE_classification_V6-2.pdf [Accessed 3 April 2018].

34 Cockcroft DW, Gault MH. Prediction of creatinine clearance from serum creatinine. Nephron 1976;16:31-41.

35 Salazar DE, Corcoran GB. Predicting creatinine clearance and renal drug clearance in obese patients from estimated fat-free body mass. Am J Med 1988;84:1053-60.

36 Charlson ME, Pompei P, Ales KL, et al. A new method of classifying prognostic comorbidity in longitudinal studies: development and validation. J Chronic Dis 1987;40:373-83.

37 Lea M, Mowe M, Mathiesen L, et al. Prevalence and risk factors of drug-related hospitalizations in multimorbid patients admitted to an internal medicine ward. PLoS One 2019;14:e0220071.

38 Gail MH. Applicability of sample size calculations based on a comparison of proportions for use with the logrank test. Control Clin Trials 1985;6:112-9.

39 Fine JP, Gray RJ. A proportional hazards model for the Subdistribution of a competing risk. J Am Stat Assoc 1999;94:496-509.

40 Gillespie U, Alassaad A, Henrohn D, et al. A comprehensive pharmacist intervention to reduce morbidity in patients 80 years or older: a randomized controlled trial. Arch Intern Med 2009;169:894-900.

41 Ravn-Nielsen LV, Duckert M-L, Lund ML, et al. Effect of an inhospital multifaceted clinical pharmacist intervention on the risk of readmission. JAMA Intern Med 2018;178:375-82.

42 Makowsky MJ, Koshman SL, Midodzi WK, et al. Capturing outcomes of clinical activities performed by a rounding pharmacist practicing in a team environment: the COLLABORATE study [NCT00351676]. Med Care 2009;47:642-50.

43 Ravn-Nielsen LV, Duckert M-L, Lund ML, et al. Effect of an inhospital multifaceted clinical pharmacist intervention on the risk of readmission: a randomized clinical trial. JAMA Intern Med 2018;178:375-82.

44 Baker M, Bell CM, Xiong W, et al. Do combined pharmacist and prescriber efforts on medication reconciliation reduce Postdischarge patient emergency department visits and hospital readmissions? J Hosp Med 2018;13:152-7.

45 Craig P, Dieppe P, Macintyre S, et al. Developing and evaluating complex interventions: the new medical Research Council guidance. BMJ 2008;337:a1655.

46 Richards D, Hallberg IR. Complex interventions in health. An overview of research metods. Routledge, 2015.

47 Fernandes $\mathrm{O}$. Medication reconciliation in the hospital: what, why, where, when, who and how? Healthc Q 2012;15 Spec No:42-9.

48 Griese-Mammen N, Hersberger KE, Messerli M, et al. PCNE definition of medication review: reaching agreement. Int J Clin Pharm 2018;40:1199-208. 